\title{
The Functional, Communicative, and Critical Health Literacy (FCCHL) Scales: Cross-Cultural Adaptation and the Psychometric Properties of the Iranian Version
}

\author{
Mahnoush Reisi, ${ }^{1}$ Firoozeh Mostafavi, ${ }^{1}$ Homamodin Javadzede, ${ }^{2}$ Behzad Mahaki, ${ }^{3}$ Gholamreza \\ Sharifirad, ${ }^{4, *}$ and Elahe Tavassoli ${ }^{5}$ \\ ${ }^{1}$ Department of Health Education and Health Promotion, School of Health, Isfahan University of Medical Sciences, Isfahan, IR Iran \\ ${ }^{2}$ Department of Health Education and Health Promotion, School of Health, Bushehr University of Medical Sciences, Bushehr, IR Iran \\ ${ }^{3}$ Department of Bio-statistics and Epidemiology, School of Health, Isfahan University of Medical Sciences, Isfahan, IR Iran \\ ${ }^{4}$ Department of Public Health, School of Health, Qom University of Medical Sciences, Qom, IR Iran \\ ${ }^{5}$ Department of Public Health, School of Health, Shahrekord University of Medical Sciences, Shahrekord, IR Iran \\ "Corresponding author: Gholamreza Sharifirad, Department of Public Health, School of Health, Qom University of Medical Sciences, Qom, IR Iran. Tel: +98-9131290174, E-mail: \\ sharifirad@hlth.mui.ac.ir
}

Received 2015 May 09; Revised 2015 July 02; Accepted 2015 August 08.

\begin{abstract}
Background: Patients today are required to understand more and more complex health information, and to navigate increasingly complex health systems. As a result, they need to develop skills such as finding, processing, understanding, and applying information about health issues, which has been conceptualized as health literacy (HL). Assessing HL is critical to providing meaningful health information to patients.

Objectives: This study aimed to examine the cultural adaptation and the psychometric properties of the Iranian version of the functional, communicative and critical health literacy (FCCHL) scales measuring three aspects of HL among type 2 diabetic patients. Patients and Methods: We conducted a methodological survey of 187 patients with type 2 diabetes using a cross-sectional design. The study was carried out in two phases: the first phase was designed to obtain a cross-cultural equivalent of the FCCHL scales, based on Beaton's guidelines. In the second phase, a cross-sectional study was conducted to evaluate the psychometric properties of the questionnaire.

Results: Exploratory factor analysis (EFA) identified three main factors with 27.07\%, 22.46\%, and 16.23\% of extracted variance, respectively. Confirmatory factor analysis (CFA) completely supported the three-factor model of the HL scales. Internal consistency was approved for the total scale $(\alpha=0.82)$ and for the functional, communicative, and critical subscales $(\alpha=0.91,0.80$, and 0.76 , respectively). Convergent validity analysis indicated a significant positive correlation $(r=0.45 ; \mathrm{P}<0.01)$ between the scores on the functional HL scales and the Iranian version of the Short Test of Health Literacy in Adults (S-TOFHLA), which was to be expected.

Conclusions: We concluded that the FCCHL scales are valid and reliable, and can be used to measure health literacy among Iranian diabetic patients. However, further research is needed to establish stronger psychometric properties for the use of this questionnaire in Iran.
\end{abstract}

Keywords: Health Literacy, Validation Studies, Psychometrics, Questionnaires

\section{Background}

Although health information can increase patient knowledge and has the potential to balance communication between health-care providers and patients, the availability of information does not automatically lead to patients becoming cognizant of and more involved in the health system $(1,2)$. Nevertheless, with the ever-expanding proliferation of media reports and diffusion via the Internet, patients find themselves in a world that is full of easily accessible, though unsubstantiated, health information resources (3-5).

Therefore, an individual's skills in finding, processing, understanding, and applying information about health is- sues may have a considerable effect on their health and future well-being (6). These skills have been conceptualized as health literacy (HL) and have received increasing worldwide attention $(7,8)$. Poor HL has been linked to poor health outcomes such as poorer health knowledge (9), lower self-efficacy $(10,11)$, poorer medication adherence $(12,13)$, poorer control of chronic illness $(14,15)$, higher hospitalization rates (16), and higher health care costs $(17,18)$.

Since poor HL is often unrecognized by health providers (19) and as patients often fail to disclose their HL difficulties because they may be ashamed of their limited skills $(7,20,21)$, health workers are urged to assess the HL of their patients in order to identify patients with low HL,

Copyright (c) 2016, Iranian Red Crescent Medical Journal. This is an open-access article distributed under the terms of the Creative Commons Attribution-NonCommercial 4.0 International License (http://creativecommons.org/licenses/by-nc/4.0/) which permits copy and redistribute the material just in noncommercial usages, provided the original work is properly cited. 
and who may therefore need additional support $(22,23)$.

Reviews of current HL measures show a degree of dissatisfaction among researchers and health providers concerning the theoretical foundations of the measures (22). Measuring HL is likely to be a particular challenge (24) because no consensus has yet been achieved on how to accomplish HL skills $(25,26)$. Current instruments reveal several problems. First, the HL measures that have come under scrutiny are time-consuming and impracticable in clinical settings. Therefore, the availability of a quick and inexpensive tool to identify patients with limited HL would offer significant improvements to the assessment of $\mathrm{HL}$, even in busy clinical settings $(2,26)$. Secondly, some researchers have defined HL as being closely linked to reading, writing and numeracy skills: "being able to apply literacy skills to health-related resources such as, prescriptions, pamphlets, medicine labels, and guidelines" (27). Thus, most existing HL measures focus mainly on reading comprehension, whereas $\mathrm{HL}$ is more than a matter of functional literacy. Individuals need a broader variety of cognitive and social skills for dealing with and acting on all kinds of health information that go beyond basic reading and writing skills (28-30).

Nutbeam has introduced a detailed and concrete framework for HL, which includes three levels (29). Basic or functional HL includes reading and writing skills. These skills allow people to function more effectively in their daily lives. Communicative or interactive HL includes additional skills that allow people to extract related information from various communicative channels and use them in order to change the situation. Finally, critical HL refers to the more advanced skills that are required for information analysis and the ability to use knowledge to apply more control on life events and situations $(8,29,31)$.

Recently, Ishikawa et al. (31) have developed a selfreported HL scale that follows Nutbeam's model. Unlike existing HL instruments, this scale, which measures all three levels, looks to be a promising tool for measuring the full spectrum of HL (2). Ishikawa et al. examined the threefactor structure of $\mathrm{HL}$ in a sample of patients with diabetes (31). The FCCHL scales are simple and easy to use and have also been validated in a Dutch version (2). The use of the FCCHL application is well documented as a screening tool in research settings (32).

Considering the importance of $\mathrm{HL}$, both in terms of research and the health system, the availability of a reliable and valid instrument to measure HL is essential in order to improve delivery services in the health system.

\section{Objectives}

The objectives of this study were two-fold:
(1) To translate and adapt the English version of the FCCHL scales to the Persian language, ensuring that the translated and original versions are cross-culturally equivalent.

(2) To determine the psychometric properties of the Iranian version of the FCCHL scales.

\section{Patients and Methods}

This methodological study, which used a crosssectional design, was conducted in Isfahan, in the central region of Iran, in 2014. The study was carried out in two phases. In the first phase, we obtained a cross-culturally equivalent Iranian version of the FCCHL scales. The next phase aimed to determine the psychometric properties of the Iranian version by examining convergent validity, construct validity, and by testing internal consistency and test-retest reliability.

\subsection{First Phase (Cross-Cultural Adaptation)}

Beaton et al. have suggested a standard set of procedures for cross-cultural adaptation. Their methods cover each step of the development process: (a) forward translation; (b) synthesis of the translations; (c) back-translation; (c) use of an expert panel; and (d) testing the penultimate version (33). In the present study, the cross-cultural adaptation was performed based on these guidelines. In terms of forward translation, the English version of the questionnaire was translated to the Persian language by two bilingual translators. One of the translators was a health professional and was familiar with HL terminology, whereas the other translator was not. With regard to a synthesis of the translations, the principal author and the two translators reviewed the two initial translations. Words that were translated differently were highlighted and discussed before making a decision on the final synthesized version of the instrument. The synthesized version was then translated back to English by two independent, bilingual, and native speaking English translators who were unaware of the original English FCCHL version. An expert panel was then convened to review the current literature in order to detect and resolve any inadequate terms or concepts in the translation as well as any inconsistencies between the initial translation and the back-translated version of the questionnaire. The expert panel comprised two PhD students in health education, one nurse educator for diabetic patients, one medical doctor, the translators and two members of the research team. To quantify the extent of expert agreement, we used the content validity index (CVI). For content validity, the experts were asked to evaluate each item for simplicity, relevance, and clarity, using a 4-point Likert-type scale, with 1 being a low score and 4 a high 
score. For items rated as 4, we asked the expert who provided this rating to give his or her suggestions for modifying it. A CVI score of 0.78 or above indicates acceptable content validity (34). The result of this step was the penultimate Iranian version of the FCCHL scales. At this stage, pre-testing was conducted using a convenience sample of 30 diabetic patients (based on guidelines), who were fluent in the Persian language. We asked the respondents to complete the questionnaire and comment on words or sentences that were difficult to understand. Unclear words were discussed with members of the expert panel and the target group. According to the result of the discussion, final adjustments were made based on the subjective judgment of the research team. The mean and variance scores of each item were examined through descriptive statistics for item analysis. If the mean of an item greatly diverged from the total item mean or if its variance was near zero, that item was dropped. The outcome of the first phase of the study, described above, was a supposedly equivalent instrument in the Persian language, which had been adapted for the local culture.

\subsection{Second Phase (Psychometric Properties of the Instrument)}

The cross-cultural adaption does not indicate any equivalence of psychometric properties. In the process of translating and adapting the original questionnaire, these properties may change. Therefore, a cross-sectional study was conducted to evaluate the psychometric properties of the culturally-adapted questionnaire by examining convergent and construct validity, and also by testing internal consistency and test-retest reliability.

\subsubsection{Recruitment of Clinical Subjects}

This study was conducted between May and July in 2014. A total of 200 Iranian patients suffering from type 2 diabetes were recruited from the Hazrate-Ali diabetes clinic using the convenience sampling procedure. The Hazrate-Ali diabetes clinic is a government health center affiliated to the Isfahan University of Medical Sciences, which provides comprehensive management and education to over 1500 individuals with type 2 diabetes. The study sample size was estimated as proposed by Bentler \& Chou with a minimum of 5 subjects and a maximum of 10 for each parameter (35). The Kaiser-Meyer-Olkin (KMO) procedure was also used as a criterion for adequate sampling in the EFA. Thirteen patients were omitted from the sample because they did not meet the inclusion criteria, did not agree to participate, or did not complete the questionnaire. The final sample therefore consisted of $187 \mathrm{di}-$ abetic patients. Inclusion criteria were that patients: (1) were aged 25 years and above; (2) had been diagnosed with type 2 diabetes $\geq 1$ year; (3) had an appropriate location to answer the questions; (4) had no cognitive problems or mental disease; and (5) participated voluntarily in the study. All participants signed letters to confirm their informed consent.

\subsubsection{Measures}

Socioeconomic data included age, sex and educational level. The 14-item FCCHL scales were used to assess HL. The scales include five items each for functional and communicative HL, and four for critical HL. Each item was rated from 1 (never) to 4 (often). To obtain scores for each subscale, the scores for the items in each subscale were summed and divided by the number of constituent items. Scores were recoded for functional HL, and mean scores were calculated for each scale ranging from 1 (low HL) to 4 (high HL). Higher scores indicate higher levels of HL and, in contrast to most HL screening tools, there is no cut-off point. The FCCHL scales do not classify patients' HL levels as adequate or inadequate. The original FCCHL scales have acceptable internal consistencies of functional $(\alpha=0.84)$, communicative ( $\alpha=0.77$ ), and critical ( $\alpha=0.65)$ HL. Cronbach's $\alpha$ of the total HL scale was reported as 0.78 (8). At the time of the study, there were two valid screening tools for HL: S-TOFHLA and Newest Vital Sign (NVS) (36). Both tools measure reading and numeracy skills. We decided to use the Iranian version of S-TOFHLA to test FCCHL for convergent validity. This was because NVS is based on reading the nutrition label from an ice cream container and our target group comprised diabetic patients for whom eating ice cream is not in keeping with their dietary recommendations. The S-TOFHLA is a two-part test. The first part comprises a four-item numeracy test using medical information or labeled prescriptions. Participants read the scenarios and then answer the questions. Reading comprehension forms the second part of the test, and includes 2 prose passages and 36 items. Participants read text passages where particular words have been deleted and replaced with blank spaces. They are asked to fill in the blanks using words from a multiple-choice list. In our STOFHLA, each question in the first part was multiplied by 7 , and each item in the second part was multiplied by 2 to create a score from 0 to 28 for numeracy, and 0 to 72 for reading comprehension. The sum of the two parts produced the S-TOFHLA score, which can range from 0 to 100 (37). For the original S-TOFHLA, Cronbach's alpha was 0.68 for the 4 numeracy items, and 0.97 for the 36 items in the 2 prose passages (37). In a previous study, the Cronbach's $\alpha$ coefficient of the Iranian version of the S-TOFHLA was 0.69 for numeracy and 0.78 for reading comprehension (36). 


\subsubsection{Statistical Analysis}

All analyses were conducted using SPSS (version 16.0) and Amos Graphic (version 18.0) software. To examine the psychometric properties of the Iranian version of the FCCHL scales, several tests were used. Internal consistency was calculated using Cronbach's' alpha coefficient: an alpha score equal to or greater than 0.70 was considered acceptable. The Pearson correlation coefficient was used for test-retest reliability. Thirty subjects completed the questionnaire twice, with a two-week interval between assessments. Convergent validity was assessed by the Pearson product moment statistic (Pearson's correlation coefficient $=r$ ) between subscales of FCCHL and S-TOFHLA. Here, an $r$ equal to 0.40 or above was considered satisfactory. EFA was performed using principal axis factoring via the promax rotation method. The Kaiser-Meyer-Olkin (KMO) measure for sampling adequacy and Bartlett's test of sphericity were also used. KMO indicates the proportion of variance in variables that can be explained by underlying factors. High values ( $>0.7$ ) indicate that the sample size is adequate for data. A scree plot was used to determine the optimal number of factors. The relationships between items and factors were determined based on factor loading values of 0.3 or higher.

In addition, CFA was employed to assess the factorial validity of the FCCHL scales. The following indices were used to evaluate model fit according to the recommendations of Hu et al. (38): the comparative fit index (CFI), the Tucker-Lewis index(TLI), the root mean squared error of approximation (RMSEA), and the normed $\chi^{2}\left(\chi^{2} / \mathrm{df}\right)$ and parsimonious normed fit index (PNFI). The following cut-offs were used for acceptable fit: CFI $\geq 0.90$, TLI $\geq 0.90$, RMSEA $\leq 0.08$, normed $\chi^{2}<5$, and PNFI $\geq 0.50$ (38).

\subsubsection{Ethical Considerations}

Ethical approval was received from the Isfahan University of Medical Sciences (ID:393268, Date: May 10, 2014) and the Isfahan provincial deputy of health. The aims and procedures of the study were explained to the participants. The anonymity and confidentiality of the study were assured and the participants then signed informed consent letters. The investigators guaranteed that there were no conflicts of interest.

\section{Results}

The characteristics of the sample and the HL scores are described in Table 1. The mean score was the highest on the communicative subscale and the lowest on the functional subscale. As functional HL assesses whether a participant has adequate reading and writing skills to understand and follow basic health information, these results indicate that the diabetic patients in the sample probably had difficulties with written material. Age was negatively associated with functional $(\mathrm{r}=-0.15, \mathrm{P}<0.05)$ and critical $(\mathrm{r}=-.19$, $\mathrm{P}<0.01) \mathrm{HL}$. A low level of HL in functional, communicative and critical $(\mathrm{P}<0.001)$ subscales was found to be more prevalent in participants who had a low educational attainment. Sex was not associated with HL levels.

\begin{tabular}{lc} 
Table 1. The Characteristics of the Respondents and Descriptive Findings $(\mathrm{n}=187)^{\mathrm{a}}$ \\
\hline Characteristics & Values \\
\hline Age, $\mathbf{y}$ & $57.4 \pm 11.07$ \\
\hline Range & $28-84$ \\
\hline Sex, No. (\%) & $127(67.9)$ \\
\hline Women & $60(32.1)$ \\
\hline Men & \\
\hline Education, No. (\%) & $45(24.1)$ \\
\hline Illiterate & $107(57.2)$ \\
\hline Primary/secondary schooling & $11(5.9)$ \\
\hline High school & $24(12.8)$ \\
\hline Above high school & $1.98 \pm 0.95$ \\
\hline Functional HL & $2.44 \pm 0.74$ \\
\hline Communicative HL & $2.43 \pm 0.82$ \\
\hline Critical HL & $2.28 \pm 0.57$ \\
\hline Total HL & $34.24 \pm 19.27$ \\
\hline S-TOFHLA & \\
\hline
\end{tabular}

${ }^{\mathrm{a}}$ Values are expressed as mean (SD) unless otherwise indicated.

\subsection{First Phase (Cross-Cultural Adaptation)}

There were no discrepancies between translations, and all items were retained for expert review. In qualitative evaluation of the tool, based on an expert panel, some adaptations were made to the wording of four items in the FCCHL scales. Item 1 in the functional subscale, which read "found that the print was too small to read", was modified to: "found that the words of the print were too small to read". Item 4 , in the communicative subscale, which read "communicated your thoughts about your illness to someone", was modified to: "communicated about your illness to someone". Item 5 , in the communicative subscale, which read "applied the obtained information to your daily life", was modified to: "applied the obtained information about your disease to your daily life". Item 4, in the critical subscale, which read "collected information to make health-related decisions", was modified to: "collected sufficient information to make health-related deci- 
sions". Experts also provided written feedback on the clarity, simplicity and relevance of the content of FCCHL items. The average CVI of the Iranian version of the FCCHL was 0.82 .

In pre-testing, we asked patients to complete the questionnaire and remark on words or sentences that were tough to comprehend. We also asked patients to suggest words or phrases that could be used to avoid misunderstanding or confusion. Various problems were encountered by the participants, mostly with regard to the critical subscale. Most patients found it difficult to understand item 1 in the critical subscale. They often failed to understand what was meant by "applicable to your situation". Some examples were provided to enable participants to understand this item. Patients also had problems with items 2 and 3 in the critical subscale. They commented that answering these questions depended on the information sources available. Patients reported that they query the credibility, validity, and reliability of the information that they receive from sources such as the Internet or friends, but that they usually trust information from physicians, nurses or other health care providers. Therefore, we stated that this question was about sources other than health care professionals. A few other points were mentioned by participants about the wording of other questions. These were all discussed with members of the expert panel and, according to the results of the discussion, final adjustments were made based on the subjective judgment of the research team.

Based on item analysis results, the range of the mean score was from 1.84 to 2.65 , and the range of the variance score was from 0.88 to 1.21 . The item-total correlations were all positive and ranged from 0.84 to 0.89 for functional $\mathrm{HL}$, from 0.73 to 0.82 for communicative HL, and from 0.71 to 0.78 for critical HL. There was no evidence of floor or ceiling effects, and the value of the skewness for the items was mostly below one.

\subsection{Internal Consistency and Test-Retest Reliability}

Cronbach's alpha coefficient was acceptable $(\alpha=0.82)$ for all items on the FCCHL scales. It was also acceptable for functional, communicative, and critical items ( $\alpha=0.91$, 0.80 , and 0.76 , respectively). The test-retest reliability coefficient was $0.85(\mathrm{P}<0.01)$, which indicates that the instrument is stable over time.

\subsection{Convergent Validity}

Pearson's correlation coefficient revealed a moderately positive association $(\mathrm{r}=0.45 ; \mathrm{P}<0.01)$ between the scores on the functional HL and the Iranian version of the $S$ TOFHLA. A moderate correlation $(\mathrm{r}=0.30 ; \mathrm{P}<0.01)$ was also found between the communicative HL scale and the $S$ TOFHLA. Non-significant small correlations were found between critical HL and the S-TOFHLA. Correlations between both total scores on the FCCHL scales and the S-TOFHLA were weak to moderate $(r=0.16 ; \mathrm{P}<0.04)$.

\subsection{Construct Validity}

Principal axis factoring via the promax rotation method was performed in order to obtain the best fitting structure and the correct number of factors. In this step of the EFA, the KMO index was equal to 0.821, and the results of Bartlett's sphericity test were significant in the confidence interval of $95 \%\left(\chi^{2}=1606, \mathrm{df}=91, \mathrm{P}<0.001\right)$. As expected, the structure of three distinct domains is clearly present. The analysis revealed three factors with an eigenvalue $>1$, which jointly accounted for $65.77 \%$ of the total sample variance (Table 2). The scree plot also indicated the three factors solution (Figure 1). The promax rotation showed the three factors emerging from a total of 14 items, all loaded above 0.40. All five functional and communicative HL items loaded on these components, but similar to the original version of the FCCHL, the first question of the critical HL subscale loaded on both the second and third components, with the difference that, in our study, factor loading on the second component is almost high. The results are shown in Table 3.

Table 2. The Results of Principal Component Factor Analysis for FCCHL

\begin{tabular}{|c|c|c|c|}
\hline Factors & Eigenvalue $^{\mathrm{a}}$ & $\begin{array}{l}\text { Percentiles of } \\
\text { Variance }^{\text {b }}\end{array}$ & $\begin{array}{l}\text { Cumulative } \\
\text { Percentiles }^{b}\end{array}$ \\
\hline Functional HL & 4.45 & 27.07 & 27.07 \\
\hline Critical HL & 3.73 & 22.46 & 49.53 \\
\hline $\begin{array}{l}\text { Communicative } \\
\text { HL }\end{array}$ & 1.1 & 16.23 & 65.77 \\
\hline
\end{tabular}

${ }^{\mathrm{a}}$ The latent dimension is usually taken to be equal to the number of eigenvalues that are $>1.0$.

${ }^{\mathrm{b}}$ Explained variation given inclusive of this factor

Based on the results of the EFA, the hypothesized measurement model included functional HL as a latent variable with five items as indicators, communicative HL as a latent variable with five items as indicators, and critical HL as a latent variable with four items as indicators. CFA results indicated that the data had acceptable fit indices for a three-factor model of the HL scales. The results are shown in Table 4.

\section{Discussion}

In this ever-changing and developing era of science and practice, the need for patients to be "health literate" 
Table 3. Rotated Factor Matrix of the FCCHL

\begin{tabular}{|c|c|c|c|}
\hline Items & Factor 1 & Factor 2 & Factor 3 \\
\hline \multicolumn{4}{|l|}{ Functional $\mathrm{H}$} \\
\hline \multicolumn{4}{|l|}{ In reading instructions or leaflets from hospitals/pharmacies, you: } \\
\hline 3. Found that the content was too difficult & 0.899 & & \\
\hline 2. Found characters and words that you did not know & 0.890 & & \\
\hline 4. Needed a long time to read and understand the content & 0.870 & & \\
\hline 1. Found that the print was too small to read & 0.834 & & \\
\hline 5. Needed someone to help you read the content & 0.831 & & \\
\hline \multicolumn{4}{|l|}{ Communicative HL } \\
\hline \multicolumn{4}{|l|}{ Since being diagnosed with diabetes, you have: } \\
\hline 5. Applied the obtained information to your daily life & & 0.813 & \\
\hline 3. Understood the obtained information & & 0.770 & \\
\hline 1. Collected information from various sources & & 0.718 & \\
\hline 2. Extracted the information you wanted & & 0.679 & \\
\hline 4. Communicated your thoughts about your illness to someone & & 0.443 & \\
\hline \multicolumn{4}{|l|}{ Critical HL } \\
\hline \multicolumn{4}{|l|}{ Since being diagnosed with diabetes, you have: } \\
\hline 4. Collected information to make health-related decisions & & & 0.830 \\
\hline 2. Considered the credibility of the information & & & 0.773 \\
\hline 3. Checked whether the information was valid and reliable & & & 0.548 \\
\hline 1. Considered whether the information was applicable to your situation & & 0.646 & 0.513 \\
\hline
\end{tabular}

Table 4. Summary of Fit Indices for Confirmatory Factor Analysis of the Culturally-Adapted FCCHL $(n=187)$

\begin{tabular}{lccccccc}
\hline FCCHL & NPAR & DF & TLI & CFI & RMSEA & PNFI & $\chi^{2} /$ df \\
\hline 3 Factors & 41 & 73 & 0.93 & 0.92 & 0.077 & 0.76 \\
\hline
\end{tabular}

Abbreviations: CFI, Comparative Fit Index; DF, Degrees of Freedom; PNFI, Parsimony Normed Fit Index; RMSEA, Root Mean-Square Error of Approximation; TLI, TuckerLewis Index; $\chi^{2} / \mathrm{DF}$, Normed $\chi^{2}$.

is stronger than before. Nowadays, patients need to participate in more complex self-care regimes, must be able to comprehend multifaceted health information, and find their way around a complex health system (39). Therefore, it is critical to assess a patient's HL in order to offer additional support and easy-to-understand health information based on the patient's HL level. In this regard, the purpose of this study was to provide a reliable and valid instrument for use in both research and in health systems in Iran.

First, a multi-stepped translation and back-translation process was undertaken during the development of the Iranian version of the FCCHL scales. Although the procedures were carefully designed, based on Beaton's guidelines, we could not ensure that the translation was adequate because of the differences in language and culture.
However, we asked the translators to emphasize conceptual rather than literal translation and also to consider the typical respondent for the instrument. We also asked them to provide all reasonable options and to think thoroughly about problematic question items and response choices before the expert meeting. A number of items with problematic words or phrases that did not completely capture the concept were identified and resolved. A complete translated version of the FCCHL scales was produced, and the content validity of the questionnaire was supported based on the evaluation of the expert committee.

According to patients' comments, some items on the FCCHL scale were changed in order to provide a more culturally appropriate wording and also to give more understandable content. Item analysis is an extra means to de- 


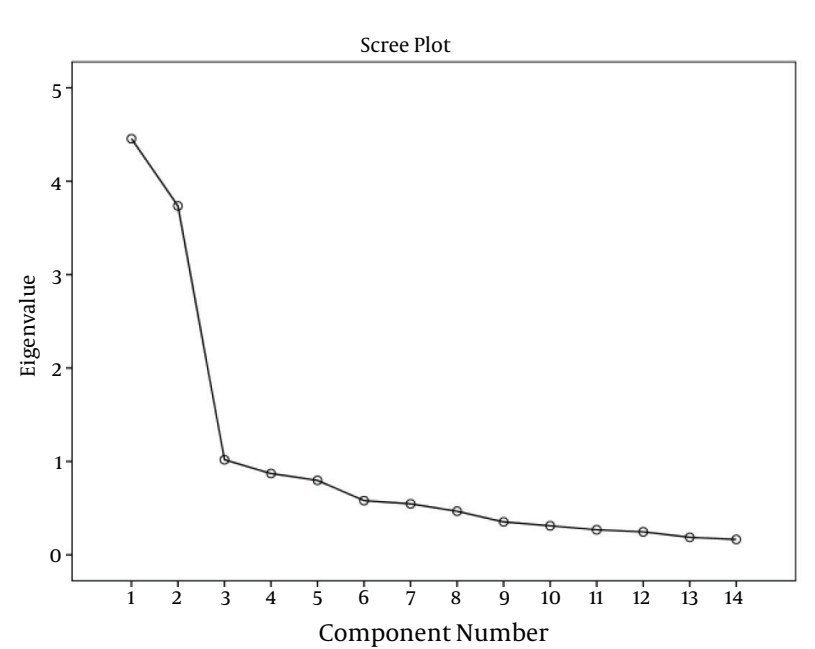

Figure 1. Scree Plot for Principal Component Analysis of the Culturally-Adapted FCCHL $(\mathrm{N}=187)$

tect weaknesses in measurements and can evaluate how well each item contributes to the whole (40). In this study, the mean and variance scores of each item, item-total correlation, confirmed that the items were homogenous to the scales.

Another important issue is whether the Iranian version of the FCCHL scales can be used for Iranian patients. Accordingly, the psychometric properties of the questionnaire were evaluated. The findings suggest that the FCCHL scales are a reliable and valid measure of HL among Iranian patients, and can be used for the assessment of a wide range of HL skills. The internal consistency of the HL scales was acceptable ( $\alpha=0.82$ ) and indicated a satisfactory degree of consistency between items for each subscale ( $\alpha=$ 0.76 to 0.91 ). Similar results have been reported for the original measure (31) and also for the Dutch version of the measure (2). The Pearson correlation coefficient also indicated good consistency of the test results over time $(\mathrm{r}=$ $0.85, \mathrm{P}<0.01)$.

The FCCHL scales have been tested for convergent validity. We examined the relationship between the FCCHL scales and the Iranian version of the S-TOFHLA two different tools measuring the same concept to provide evidence of the instrument's ability to measure the HL concept. A moderate positive correlation was found between the functional HL subscale and the Iranian version of the S-TOFHLA. On the other hand, the correlation between the FCCHL scales and the Iranian version of the S-TOFHLA was weak to moderate. This result was anticipated as the FCCHL scales assess a broader range of HL skills, including communication and critical capacities, whereas the S-TOFHLA only measures the functional concept of HL whether a par- ticipant's reading and writing skills are sufficient to comprehend and follow simple health information. These results provide evidence that the FCCHL scales successfully measure the HL concept.

The construct validity of the FCCHL scales was assessed through factor analysis. The results support a three-factor structure for the instrument and were consistent with a previous study for the Dutch version (2). All items loaded above 0.40 , and similar results were reported for the Dutch version, with standardized factor loadings ranging between 0.58 and 0.95 (2). In this study, the first question in the critical subscale ("considering whether the information was applicable to your situation") was loaded in both communicative and critical factors. In Nutbeam's framework for HL, patient skill in critically analyzing information and using that information to exert greater control over life events and situations is referred to as the critical concept of HL. This question was therefore based precisely on this definition, and we included this item in the critical HL subscale because of the theoretical definition even though factor loading on the communicative component was higher than on the critical component. The FCCHL scales accounted for $65.77 \%$ of the variance in total HL scores. Functional, communicative, and critical HL accounted for $27.07 \%, 22.46 \%$, and $16.23 \%$, respectively, of the variance. This rate was similar to results for the original questionnaire (8). The CFA results confirmed that the data had a good fit with the three-factor scale. The results of psychometric evaluation of the Dutch translation of the FCCHL scales also indicated that model's fit was good (2).

To the best of the authors' knowledge, this is the first study in which cross-cultural adaptation and the psychometric properties of the multidimensional scale for $\mathrm{HL}$ have been evaluated in Iranian patients with type 2 diabetes. This is also the first time that a version of the FCCHL scales has been tested for convergent validity using a valid questionnaire such as the S-TOFHLA. It seems that the main weakness of this study relates to the unsuccessful generalizability of results to all Iranians with type 2 diabetes. It is also possible that patients with very low HL declined to participate in this study, and we have no data on the sociodemographic characteristics of those who refused to participate. Consequently, additional research is both recommended and necessary to confirm the study findings.

In general, the current study indicates that the Iranian version of the FCCHL scales is a reliable measure for assessing HL. However, as the FCCHL scales are a recently developed measure, additional studies are needed to confirm their reliability and validity and to focus on other psychometric properties of the questionnaire. Although use of convenience sampling from one diabetes clinic may be supposed to limit the generalization of the results, the re- 
sults are nevertheless of major significance for Iranian patients with type 2 diabetes.

\section{Acknowledgments}

We would like to thank all the diabetic patients who participated in this study.

\section{Footnote}

Funding/Support: This work was supported financially by the Isfahan University of Medical Science of Iran (No.393268).

\section{References}

1. Paasche-Orlow MK, Parker RM, Gazmararian JA, Nielsen-Bohlman LT, Rudd RR. The prevalence of limited health literacy. J Gen Intern Med. 2005;20(2):175-84. doi: 10.1111/j.1525-1497.2005.40245.x. [PubMed: 15836552].

2. van der Vaart R, Drossaert $\mathrm{CH}$, Taal E, ten Klooster PM, HilderinkKoertshuis RT, Klaase JM, et al. Validation of the Dutch functional communicative and critical health literacy scales. Patient Educ Couns. 2012;89(1):82-8. doi: 10.1016/j.pec.2012.07.014. [PubMed: 22878030].

3. Napoli PM. Consumer use of medical information from electronic and paper media: A literature review. The internet and health communication. 2001:79-98.

4. Hesse BW, Nelson DE, Kreps GL, Croyle RT, Arora NK, Rimer BK, et al. Trust and sources of health information: the impact of the Internet and its implications for health care providers: findings from the first Health Information National Trends Survey. Arch Intern Med. 2005;165(22):2618-24. doi: 10.1001/archinte.165.22.2618. [PubMed: 16344419].

5. Passalacqua R, Caminiti C, Salvagni S, Barni S, Beretta GD, Carlini P, et al. Effects of media information on cancer patients' opinions, feelings, decision-making process and physician-patient communication. Cancer. 2004;100(5):1077-84. doi: 10.1002/cncr.20050. [PubMed: 14983505].

6. Gray NJ, Klein JD, Noyce PR, Sesselberg TS, Cantrill JA. The Internet: a window on adolescent health literacy.JAdolesc Health. 2005;37(3):243. doi:10.1016/j.jadohealth.2004.08.023. [PubMed:16109345].

7. Kindig DA, Panzer AM, Nielsen-Bohlman L. Health Literacy: A Prescription to End Confusion. Washington (DC): National Academies Press; 2004

8. Ishikawa H, Nomura K, Sato M, Yano E. Developing a measure of communicative and critical health literacy: a pilot study of Japanese office workers. Health Promot Int. 2008;23(3):269-74. doi: 10.1093/heapro/dan017. [PubMed: 18515303]

9. Gazmararian JA, Williams MV, Peel J, Baker DW. Health literacy and knowledge of chronic disease. Patient Educ Couns. 2003;51(3):267-75. [PubMed:14630383].

10. Reisi M, Mostafavi F, Javadzade SH, Mahaki B, Sharifirad G. Assessment of Some Predicting Factors of Self-efficacy in Patients with Type 2 Diabetes. Iran J Endocrinol Metab. 2015;17(1):44-52.

11. Cavanaugh K, Huizinga MM, Wallston KA, Gebretsadik T, Shintani A, Davis D, et al. Association of numeracy and diabetes control. Ann Intern Med. 2008;148(10):737-46. [PubMed: 18490687].

12. Wolf MS, Davis TC, Osborn CY, Skripkauskas S, Bennett CL, Makoul G. Literacy, self-efficacy, and HIV medication adherence. Patient Educ Couns. 2007;65(2):253-60. doi: 10.1016/j.pec.2006.08.006. [PubMed: 17118617].
13. Ngoh LN. Health literacy: a barrier to pharmacist-patient communication and medication adherence. J Am Pharm Assoc (2003). 2009;49(5):132-46. doi: 10.1331/JAPhA.2009.07075. [PubMed 19748861].

14. Tang YH, Pang SM, Chan MF, Yeung GS, Yeung VT. Health literacy, complication awareness, and diabetic control in patients with type 2 diabetes mellitus. J Adv Nurs. 2008;62(1):74-83. doi: 10.1111/j.13652648.2007.04526.x. [PubMed: 18352966].

15. Schillinger D, Grumbach K, Piette J, Wang F, Osmond D, Daher C, et al. Association of health literacy with diabetes outcomes. JAMA. 2002;288(4):475-82. [PubMed: 12132978].

16. Baker DW, Gazmararian JA, Williams MV, Scott T, Parker RM, Green $\mathrm{D}$, et al. Functional health literacy and the risk of hospital admission among Medicare managed care enrollees. Am J Public Health. 2002;92(8):1278-83. [PubMed: 12144984].

17. Weiss BD, Palmer R. Relationship between health care costs and very low literacy skills in a medically needy and indigent Medicaid population. J Am Board Fam Med. 2004;17(1):44-7.

18. Howard DH, Gazmararian J, Parker RM. The impact of low health literacy on the medical costs of Medicare managed care enrollees. Am J Med. 2005;118(4):371-7. doi:10.1016/j.amjmed.2005.01.010. [PubMed 15808134].

19. Bass P3, Wilson JF, Griffith CH, Barnett DR. Residents' ability to identify patients with poor literacy skills. Acad Med. 2002;77(10):1039-41 [PubMed: 12377684].

20. Williams MV, Davis T, Parker RM, Weiss BD. The role of health literacy in patient-physician communication. Fam Med. 2002;34(5):383-9. [PubMed: 12038721].

21. Chew LD, Bradley KA, Boyko EJ. Brief questions to identify patients with inadequate health literacy. Fam Med. 2004;36(8):588-94. [PubMed: 15343421].

22. Chinn D, McCarthy C. All Aspects of Health Literacy Scale (AAHLS) developing a tool to measure functional, communicative and critical health literacy in primary healthcare settings. Patient Educ Couns. 2013;90(2):247-53. doi: 10.1016/j.pec.2012.10.019. [PubMed: 23206659].

23. Parker R. Health literacy: a challenge for American patients and their health care providers. Health Promot Int. 2000;15(4):277-83.

24. Nutbeam D. The evolving concept of health literacy. Soc Sci Med. 2008;67(12):2072-8. doi: 10.1016/j.socscimed.2008.09.050. [PubMed: 18952344].

25. Pleasant A, McKinney J. Coming to consensus on health literacy measurement: an online discussion and consensus-gauging process. Nurs Outlook. 2011;59(2):95-106 e1. doi: 10.1016/j.outlook.2010.12.006. [PubMed: 21402205].

26. Chew LD, Griffin JM, Partin MR, Noorbaloochi S, Grill JP, Snyder A, et al. Validation of screening questions for limited health literacy in a large VA outpatient population. J Gen Intern Med. 2008;23(5):561-6. doi: 10.1007/s11606-008-0520-5. [PubMed: 18335281].

27. Parker RM, Baker DW, Williams MV, Nurss JR. The test of functiona health literacy in adults: a new instrument for measuring patients literacy skills. J Gen Intern Med. 1995;10(10):537-41. [PubMed: 8576769]

28. Jordan JE, Osborne RH, Buchbinder R. Critical appraisal of health literacy indices revealed variable underlying constructs, narrow content and psychometric weaknesses.JClin Epidemiol. 2011;64(4):366-79. doi 10.1016/j.jclinepi.2010.04.005. [PubMed: 20638235].

29. Nutbeam D. Health literacy as a public health goal: a challenge for contemporary health education and communication strategies into the 21st century. Health Promot Int. 2000;15(3):259-67.

30. Peerson A, Saunders M. Health literacy revisited: what do we mean and why does it matter?. Health Promot Int. 2009;24(3):285-96. doi: 10.1093/heapro/dap014. [PubMed: 19372101]

31. Ishikawa $\mathrm{H}$, Takeuchi $\mathrm{T}$, Yano E. Measuring functional, communicative, and critical health literacy among diabetic patients. Diabetes Care. 2008;31(5):874-9. doi:10.2337/dc07-1932. [PubMed:18299446]. 
32. Lai AY, Ishikawa H, Kiuchi T, Mooppil N, Griva K. Communicative and critical health literacy, and self-management behaviors in endstage renal disease patients with diabetes on hemodialysis. Patient Educ Couns. 2013;91(2):221-7. doi: 10.1016/j.pec.2012.12.018. [PubMed: 23357415].

33. Beaton D, Bombardier C, Guillemin F, Ferraz MB. Recommendations for the cross-cultural adaptation of health status measures. New York: American Academy of Orthopaedic Surgeons; 2002.

34. Lynn MR. Determination and quantification of content validity. Nurs Res. 1986;35(6):382-5. [PubMed: 3640358].

35. Bentler PM, Chou CP. Practical issues in structural modeling. Socio Meth Res. 1987;16(1):78-117.
36. Javadzade HSG, Reisi M, Tavassoli E, Rajati F. Health Literacy among Adults of Isfahan, Iran. J Health Syst Res. 2013;9(5):540-9.

37. Baker DW, Williams MV, Parker RM, Gazmararian JA, Nurss J. Development of a brief test to measure functional health literacy. Patient Educ Couns. 1999;38(1):33-42. [PubMed:14528569].

38. Hu LT, Bentler PM. Cutoff criteria for fit indexes in covariance structure analysis: Conventional criteria versus new alternatives. Struct Equ Modeling. 1999;6(1):1-55

39. Johnson A. Health literacy, does it make a difference?. Aust J Adv Nurs. 2014;31(3):39-45.

40. Kim MT, Han H. Cultural considerations in research instrumentation development. Inst clinic health-care res. 2004:73-80. 